\title{
Correction to: Comparison of noninvasive continuous arterial blood pressure measured by NICAP with arterial line in elderly patients
}

Zhao Xu ${ }^{1}$, Hongyang Chen ${ }^{1}$, Hongyu Zhou ${ }^{1}$, Xiaohui Sun ${ }^{2}$, Jun Ren ${ }^{3}$, Hongxia Sun ${ }^{2}$, Chan Chen ${ }^{*}$ and Guo Chen ${ }^{1 *}$

Correction to: BMC Geriatr (2022) 22:108

https://doi.org/10.1186/s12877-022-02803-3

After publication of this article [1], the authors reported that the abstract contains 6 incorrect values. The original article [1] has been updated.

\section{Author details}

'Department of Anesthesiology, West China Hospital, Sichuan University, No.37 Guoxue Alley, Chengdu 610041, China. ${ }^{2}$ Department of Anesthesiology, West China Hospital, Sichuan University/ West China School of Nursing, Sichuan University, No.37 Guoxue Alley, Chengdu 610041, China. ${ }^{3}$ Department of Anesthesiology, Xinjiang Production and Construction Corps Hospital, No.

232 Qingnian Road, Urumqi 830002, China.

Published online: 28 February 2022

\section{Reference}

1. Xu Z, et al. Comparison of noninvasive continuous arterial blood pressure measured by NICAP with arterial line in elderly patients. BMC Geriatr. 2022;22:1-9. 